\title{
Computational Modeling of Visual Selective Attention Based on Correlation and Synchronization of Neural Activity.
}

\author{
Kleanthis C. Neokleous ${ }^{1}$, Marios N. Avraamides ${ }^{2}$, Christos N. Schizas ${ }^{1}$ \\ ${ }^{1}$ Department of Computer Science, ${ }^{2}$ Department of Psychology, University of Cyprus, 75 \\ Kallipoleos, 1678, POBox 20537, Nicosia, CYPRUS.
}

\begin{abstract}
Within the broad area of computational intelligence, it is of great importance to develop new computational models of human behaviour aspects. In this report we look into the recently suggested theory that neural synchronization of activity in different areas of the brain occurs when people attend to external visual stimuli. Furthermore, it is suspected that this cross-area synchrony may be a general mechanism for regulating information flow through the brain. We investigate the plausibility of this hypothesis by implementing a computational model of visual selective attention that is guided by endogenous and exogenous goals (i.e., what is known as top down and bottom-up attention). The theoretical structure of this model is based on the temporal correlation of neural activity that was initially proposed by Niebur and Koch (1994). While a saliency map is created in the model at the initial stages of processing visual input, at a later stage of processing, neural activity passes through a correlation control system which comprises of coincidence detector neurons. These neurons measure the degree of correlation between endogenous goals and the presented visual stimuli and cause an increase in the synchronization between the brain areas involved in vision and goal maintenance. The model was able to simulate with success behavioural data from the "attentional blink" paradigm (Raymond and Sapiro, 1992). This suggests that the temporal correlation idea represents a plausible hypothesis in the quest for understanding attention.
\end{abstract}

\section{Introduction}

Due to the great number of sensory stimulation that a person experiences at any given point of conscious life, it is practically impossible to integrate all information that is available to the senses into a single perceptual event. This implies that a mechanism must be present in the brain to focus selectively its resources on specific information. This mechanism, known as attention, can be described as the

Please use the following format when citing this chapter:

Neokleous, K.C., Avraamides, M.N. and Schizas, C.N., 2009, in IFIP International Federation for Information Processing, Volume 296; Artificial Intelligence Applications and Innovations III; Eds. Iliadis, L., Vlahavas, I., Bramer, M.; (Boston: Springer), pp. 215-223. 
process by which information is passed on to a higher level of processing either through relative amplification of the neural activity that represents the "to be attended" stimuli or by suppression of the distracting stimuli, or both.

Attention can be guided by top-down and bottom-up processing as cognition can be regarded as a balance between internal motivations and external stimulations. Volitional shifts of attention or endogenous attention results from "topdown" signals originating in the prefrontal cortex while exogenous attention is guided by salient stimuli from "bottom-up" signals in the visual cortex (Corbetta and Shulman, 2002).

Previous literature on attention suggests that the attention selection mechanism functions in two hierarchical stages: An early stage of parallel processing across the entire visual field that operates without capacity limitation, and a later limitedcapacity stage that deals with selected information in a sequential manner. When items pass from the first to the second stage of processing, they are typically considered as selected. (Treinsman and Gelade 1980).

Previous research suggests that attention is based on two processes. The first is known as "biased competition" (Moran and Desimone, 1985) and it is supported by findings from studies with single-cell recordings. These studies have shown that attention enhances the firing rates of the neurons that represent the attended stimuli and suppresses the firing rates of the neurons that encode the unattended stimuli. The second process, which refers to the synchronization of neural activity during the deployment of attention, is supported by studies showing that neurons selected by attention have enhanced gamma-frequency synchronization (Gruber et al., 1999; Steinmetz et al., 2000; Fries et al., 2001). For example, in a study by Fries et al. (2001) the activity in area V4 of the brain of macaque monkeys was recorded while the macaques attended relevant stimuli. Results showed increased gamma frequency synchronization for attended stimuli compared to the activity elicited by distractors. A recent study by Buelhman and Deco (2008) provided evidence that attention is affected by both biased competition and the synchronization of neural activity.

A computational model for biased competition has been proposed by Deco and Rolls (2005). In this model Deco and Rolls have shown that competition between pools of neurons combined with top-down biasing of this competition gives rise to a process that can be identified with attention. However, it should be pointed out that this model only considered rate effects while gamma synchronization was not addressed.

In the present report, we propose a computational model for endogenous and exogenous visual attention that is based on both the rate and the synchronization of neural activity. The basic functionality of the model relies on the assumption that the incoming visual stimulus will be manipulated by the model based on the rate and temporal coding of its associated neural activity. The rate associated with a visual stimulus is crucial in the case of exogenous attention since this type of attention is mainly affected by the different features of the visual stimuli. Stimuli with more salient features gain an advantage for passing through the second stage of processing and subsequently for accessing working memory. On the other hand, 
endogenous or top-down attention is mainly affected by the synchronization of incoming stimuli with the goals that guide the execution of a task. These goals are most likely maintained in the prefrontal cortex of the brain. The presence of a closed link between endogenous attention with synchronization is supported by many recent studies (Niebur et al 2002, Gross et al 2004). For example, Saalmann et al (2007) recorded neural activity simultaneously from the posterior parietal cortex as well as an earlier area in the visual pathway of the brain of macaques during the execution of a visual matching task. Findings revealed that there was synchronization of the timing activities in the two regions when the monkeys selectively attended to a location. Thus, it seems that parietal neurons which presumably represent neural activity of the endogenous goals may selectively increase activity in earlier sensory areas. In addition, the adaptive resonance theory by Grossberg (1999) implies that temporal patterning of activities could be ideally suited to achieve matching of top-down predictions with bottom-up inputs, while Engel et al (2001) in their review have noted that "If top-down effects induce a particular pattern of subthreshold fluctuations in dendrites of the target population, these could be 'compared' with temporal patterns arising from peripheral input by virtue of the fact that phase-shifted fluctuations will cancel each other, whereas inphase signals will summate and amplify in a highly nonlinear way, leading to a salient postsynaptic signal" (p.714). Finally, it should be noted that Hebbian learning suggests that action potentials that arrive synchronously at a neuron summate to evoke larger postsynaptic potentials than do action potentials that arrive asynchronously; thus, synchronous action potentials have a greater effect at the next processing stage than do asynchronous action potentials.

A mechanism for selective attention based on the rate and synchronization of the neural activity for incoming stimuli is thus used in the proposed model. The model has been implemented computationally to simulate the typical data from "the attentional blink" phenomenon (Raymond and Sapiro,1992).

\section{The Attentional Blink Phenomenon}

The Attentional Blink (AB) is a phenomenon observed with using the rapid serial visual presentation (RSVP) paradigm. In the original experiment by Raymond and Shapiro (1992), participants were requested to identify two letter targets T1 and T2 among digit distractors with each stimulus appearing for about 100ms (Figure 1.a). Results revealed that the correct identification of $\mathrm{T} 1$ impaired the identification of T2 when T2 appeared within a brief temporal window of 200-500 ms after T1. When T2 appeared outside this time window it could be identified normally (Figure 1.b series 1.).

Another important finding from the $\mathrm{AB}$ task is that when $\mathrm{T} 1$ is not followed by a mask/distractor, the $\mathrm{AB}$ effect is significantly reduced. That is, if the arrival of the incoming stimulus at $\mathrm{t}=200 \mathrm{~ms}$ (lag 2$)$ and/or lag 3 ( $\mathrm{t}=300 \mathrm{~ms})$ are replaced by a blank then the $\mathrm{AB}$ curve takes the form shown by series 2 and 3 in Figure 1. 


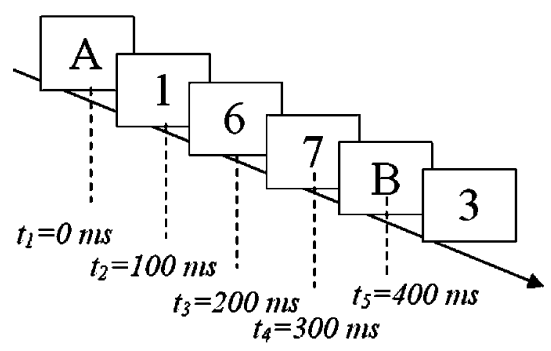

a

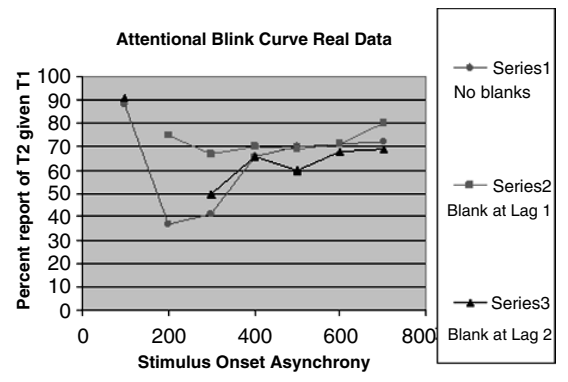

b

Figure1. Presentation of the RSVP for the "attentional blink" experiment (Figure1.a) and the typical attentional blink curve with no blanks (red series), with blank at lag 1 (green series) and blank at lag 2 (black series) based on the data of Raymond and Sapiro (1992) (Figure1.b).

\section{Neural Correlates of the Attentional Blink Phenomenon}

Event-related potentials (ERPs) are signals that measure the electrical activity of neuronal firing in the brain relative to events such as the presentation of stimuli. Over the years a number of ERP components related to attention have been identified in the literature.

The first distinguishable physiological signals are observed around $130-150 \mathrm{~ms}$ post stimulus (P1/N1 signals). Most likely, these signals correspond to the initial processing in the visual cortex and reflect early pre-frontal activation by the incoming visual stimuli. At about $180-240 \mathrm{~ms}$ post-stimulus the P2/N2 signals are observed which have become clearer over the last years with the use of MEG (Ioannides and Taylor, 2003). These signals have been proposed as control signals for the movement of attention (Hopf et al., 2000 , Taylor 2002). More specifically, the CODAM model of attention that is proposed by Taylor (2002) follows a control theory approach and uses the N2 signal as the signal from the controller that modulates the direction of the focus of attention. Moreover, in Bowman and Wyble's (2007) Simultaneous Type Serial Token $\left(\mathrm{ST}_{2}\right)$ model, when the visual system detects a task-relevant item, a spatially specific Transient Attentional Enhancement (TAE), called the blaster, is triggered. In the $\mathrm{ST}_{2}$ model the presence of a correlation between the blaster and a component of the P2/N2 signal is also hypothesized. The P300 ERP component which is present at about 350-600 ms poststimulus is taken to be an index of the availability for report of the attentionamplified input arriving from earlier sensory cortices to the associated working memory sensory buffer site. Thus, access to the working memory sensory site is expected to occur in the specific time window. Finally, the N4 component which is recorded at around $400 \mathrm{~ms}$ is related to semantic processing indicating perceptual awareness. 
The chronometric analysis of the ERPs occurring during the attentional blink has revealed some important observations. More importantly in the case where the second target was not perceived, the P1/N1 and the N400 components which are considered indices of semantic processing were still obtained even though the N2 and P300 were no longer observed (Sergent et al 2005). Thus, one possible explanation for the classic U-shaped curve of Figure 1.b (series 1) based on the identification of the second target to have a minimum at around $300 \mathrm{~ms}$, is that an early attention processing component of the second target (possibly N2 of T2) is inhibited by a late component of the first target (P300 of T1), (Vogel et al 1998,Fell et al 2001).

\section{Proposed Model}

The proposed model is a two stage model that, in contrast to other computational models, contains a correlation control module (Figure 3). That is, in the case of endogenous attention tasks, the functioning of the model is based on the synchronization of incoming stimuli with information held in the endogenous goals module which has probably been initialized by information from long -term memory (Engel et al 2001).

In the conducted simulations each stimulus that enters the visual field, is coded by determining the rate of the related neuron spikes (enhanced relatively by the salience filters) as well as the exact timing of the spikes. This means that both of these characteristics are considered in the race between the different visual stimuli to access working memory as initially implemented in a computational model by Niebur and Koch (1994).

As shown in Figure 3, a visual stimulus initially moves from the inputs module into the first stage of parallel processing. In this stage, competition among all stimuli, implemented as lateral inhibition, exerts the first impact on each of the neural responses. Following that, as the neural activity continues up through the visual hierarchy, the information from the visual stimuli passes through the semantic correlation control module. During this stage of process, a coincidence detection mechanism similar to the procedure discussed by Mikula and Niebur (2008) measures the degree of correlation between the visual stimuli and the endogenous goals (in the case of top-down attention).

This procedure provides an advantage (in the case of amplification) to the selected neural activity for accessing working memory. However, the initialization of a signal by the correlation control module (that can be implied to be relevant with the N2pc signal -component of N2/P2), can be represented by the combined firing of a neural network. Thus, it is appropriate to consider a relative refractory period each time the correlation control module "fires" or activates the specific signal for amplification or inhibition. Consequently, the refractory period of the correlation control module combined with the lateral inhibition between the RSVP items causes the attenuation of the attentional blink in the case in which the dis- 
tractors are replaced by blanks and both these mechanisms are inherited in the proposed model (series 2 and 3 in Figure1.b.).

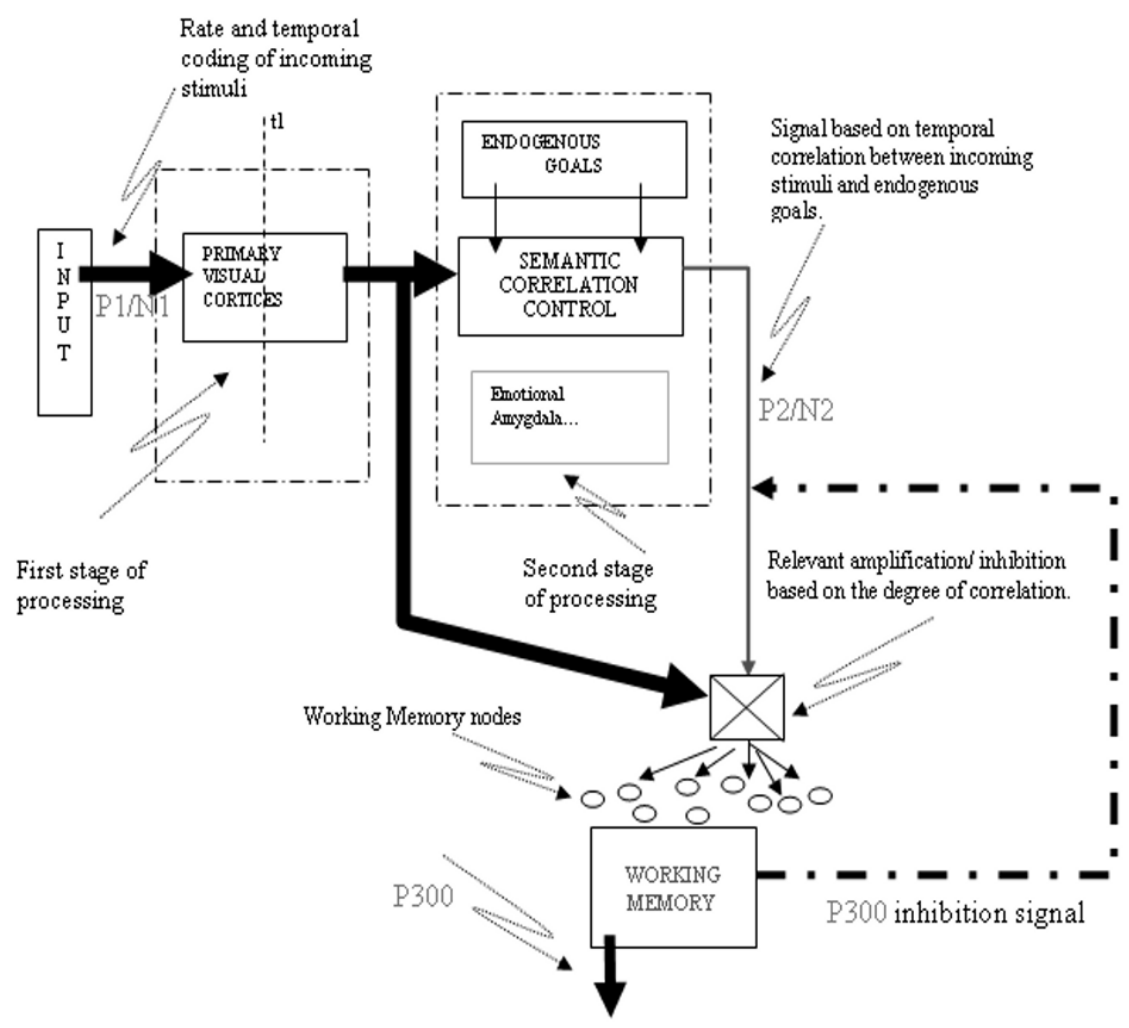

Figure 3. Diagram of the proposed computational model.

Finally, after the handling of the neural activity of each incoming stimulus, a specific working memory node is excited producing inhibition towards the other working memory nodes. After a specific threshold is passed, the working memory node will fire an action potential simulating the initialization of the P300 signal representing perceptual awareness of the specific visual stimuli as well as inhibition of the following signal from the correlation control model ( possibly the N2/ P2 signals of the following stimulus) if it appears during that specific timing.

It should be also noted that even stimuli with completely no correlation with the endogenous goals could gain access to working memory sites, provided that their response has been enhanced sufficiently by the salience filters at the first stages of processing. Thus, the model allows for exogenous shifts of attention. 


\section{Simulations and Results}

The computational model has been implemented in the Matlab-Simulink environment. Each of the visual stimuli has been represented by a $10 \mathrm{~ms}$ sequence of spikes. As seen in Figure 4, in each ms a value of one (spike) or zero (no-spike) is possible. For coding a target, a specific pattern has been decided so that if the incoming stimulus represents a target, it will have a 0.9 possibility for each time step to have the correct information. On the other hand if the incoming stimulus represents a distractor, it will have a 0.85 possibility of not having the correct information at each time step. Both distractors and targets will have the same rate, which equals to 10 spikes \pm a random noise, since both (targets and distractors) have the same effect from the salience filters (same brightness, intensity etc.).

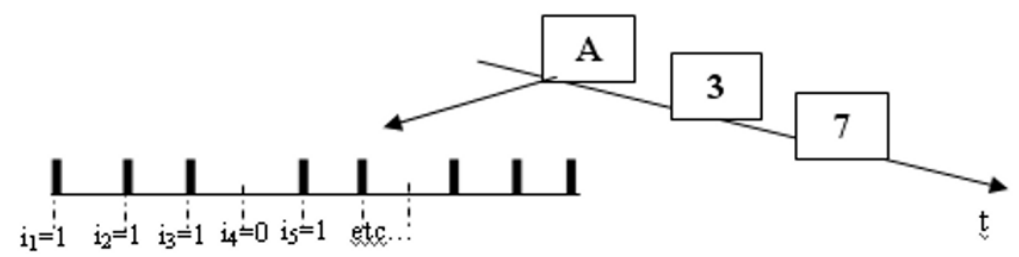

Figure4. Coding of the incoming visual stimuli.

Inside the endogenous goals module, the pattern representing the targets is saved. Therefore, when a visual stimulus enters, a coincidence detector mechanism measures the degree of correlation and fires a relative signal. For the simulations, T1 was always presented at time $t=0$ and T2 at each of the following time lags. For each time lag that T2 was presented, the simulations where run for 50 times for the three different cases. That is, when distractors capture all the available positions causing masking to the targets, with blank at lag 1 and with blank at lag 2. The simulation results compared to experimental results can be seen in Figure 5 below.
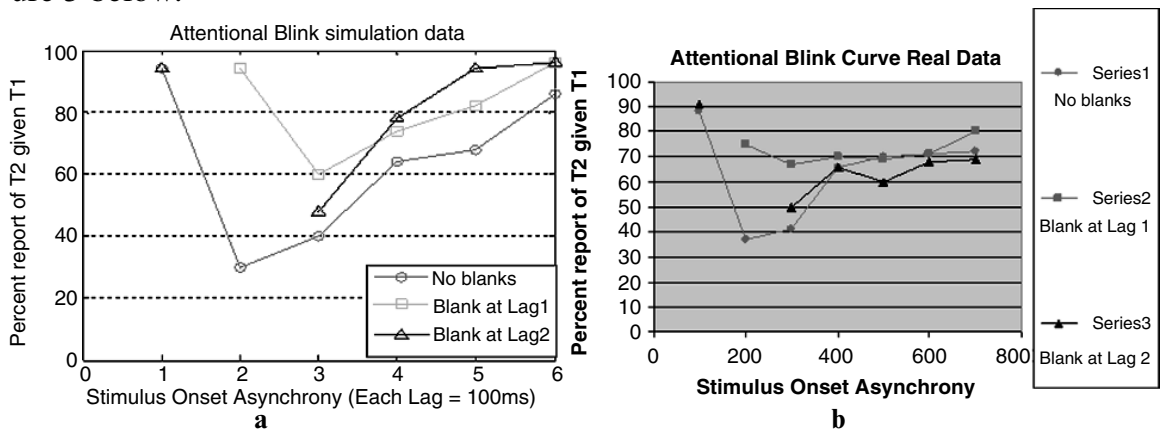

Figure5. Comparison between simulation data (5.a) and experimental data (5.b). 


\section{Discussion}

The model described above has implemented computationally a novel conjecture put forward by Niebur and Koch 1994. Niebur and Koch have suggested that imposing a temporal modulation on attended sensory signals is a plausible mechanism for producing unique percepts within the highly distributed architecture of the cortex.

The coincidence detector mechanism proposed as a basic functionality of the correlation control module between the incoming stimuli and the endogenous goals can cause an increase in the synchronization of the different cortical areas involved in the process. Actually, coincidence detector neurons in the brain are neurons that they fire if they receive synchronous inputs from other neurons. Coincidence of firing between two or more neurons can cause increase in the strength of the following synapses connected on the specific neurons. This will gradually cause synchronization between the involved brain areas as has been observed (Saalmann et al 2007) and mentioned in the previous section.

The model presented here has successfully managed to simulate the behavioral data of the attentional blink experiment giving one supplementary confirmation that the temporal correlation between different cortical areas might be an important mechanism for regulating information through the brain. Furthermore, the coincidence detector neural network model of selective attention can be used to simulate some other important attentional phenomena contributing thus to the formulation of more explicit theories of attention.

\section{References}

1. Bowman H.,Wyble S.(2007). "The Simultaneous Type, Serial Token Model of Temporal Attention and Working Memory." Psy. Re., Vol. 114

2. Buehlmann A., Deco G (2008). "The Neuronal Basis of Attention: Rate versus Synchronization Modulation". The Jour. of Neuros. 28(30)

3. Corbetta, M., Shulman, G.L. (2002)."Control of goal-directed and stimulus-driven attention in the brain". Nature R. Neuroscience 3:201-215.

4. Deco G, Rolls ET (2005). "Neurodynamics of biased competition and cooperation for attention: a model with spiking neurons". J. Neurophysi.94

5. Engel A. K., Fries P., Singer W.(2001) "Dynamic predictions: Oscillations and synchrony in top-down processing" Nature, Volume 2 pp.704-716

6. Fries P, Reynolds JH, Rorie AE, Desimone R (2001). "Modulation of oscillatory neuronal synchronization by selective visual attention". Science 291:1560-1563.

7. Grossberg, S. (1999). "The link between brain learning, attention, and consciousness". Conscious. Cogn 8, 1-44

8. Gross J., Schmitz F., Schnitzler I. et al (2004). "Modulation of long-range neural synchrony reflects temporal limitations of visual attention in humans." PNAS August 31, 2004 vol. 101 no. 35 pp13050-13055

9. Gruber T, Muller MM, Keil A, Elbert T (1999). "Selective visual-spatial attention alters induced gamma band responses in the human EEG". Clin Neurophysiol 110:2074-2085. 
10. Hopf, J.-M., Luck, S.J., Girelli, M., Hagner, T., Mangun, G.R., Scheich, H., Heinze, H.J., (2000). "Neural sources of focused attention in visual Search". Cereb. Cortex 10, 1233-1241.

11. Ioannides, A.A., Taylor, J.G., (2003). "Testing models of attention with MEG”. In: Proceedings IJCNN'03. pp. 287-297.

12. Mikula S., Niebur E., (2008). "Exact Solutions for Rate and Synchrony in Recurrent Networks of Coincidence Detectors." Neural Computation.20

13. Moran J, Desimone R (1985). "Selective attention gates visual processing in the extrastriate cortex". Science 229:782-784.

14. Niebur E., Hsiao S.S., Johnson K.O., (2002) "Synchrony: a neuronal mechanism for attentional selection?" Cur.Op. in Neurobio., 12:190-194

15. Niebur E, Koch C (1994). "A Model for the Neuronal Implementation of Selective Visual Attention Based on Temporal Correlation Among Neurons". Journal of Computational Neuroseience 1, 141-158.

16. Raymond JE, Shapiro KL, Arnell KM (1992). "Temporary suppression of visual processing in an RSVP task: an attentional blink?". J.of exp. psyc. Human perc, and performance 18 (3): 849-60

17. Saalmann Y.B., Pigarev I.N., et al. (2007). "Neural Mechanisms of Visual Attention: How Top-Down Feedback Highlights Relevant Locations" Science 3161612

18. Sergent C., Baillet S. \& Dehaene S. (2005). "Timing of the brain events underlying access to consiousness during the attentional blink." Nat Neurosci, Volume 8, Number 10, page 1391-1400.

19. Steinmetz PN, Roy A, et al.(2000). "Attention modulates synchronized neuronal firing in primate somatosensory Cortex". Nature 404:187-190.

20. Taylor J.G., Rogers M. (2002). "A control model of the movement of attention". Neural Networks 15:309-326

21. Treisman, A., \& Gelade, G. (1980). “A feature-integration theory of attention”. Cognitive Psychology, 12, 97-136.

22. Vogel E.K., Luck S.J., Shapiro K.L., (1998). "Electrophysiological evidence for a postperceptual locus of suppression during the attentional blink." J. Exp. Psychol. Hum. Percept. Perform. 24 pp.1656-1674. 